\title{
PERSPECTIVE
}

\section{THROUGH AND BEYOND COVID-19 PANDEMIC: A NEW SCENARIO FOR CARDIONCOLOGY}

\author{
L. Tarantini ${ }^{1}$, A. Camerini ${ }^{2}$, M. L. Canale ${ }^{3}$, I. Bisceglia ${ }^{4}$, D. Gabrielli ${ }^{5}$, \\ F. Colivicchi ${ }^{6}$, M. M. Gulizia ${ }^{7,8}{ }^{,}$A. Navazio ${ }^{1}$ \\ ${ }^{1}$ Department of Cardiology, Presidio Ospedaliero - Santa Maria Nuova, AUSL RE IRCCS, Reggio Emilia, Italy \\ ${ }^{2}$ Medical Oncology, Azienda USL Toscana Nord-Ovest, Versilia Hospital, Lido di Camaiore, Lucca, Italy \\ ${ }^{3}$ Division of Cardiology, Azienda USL Toscana Nord-Ovest, Versilia Hospital, Lido di Camaiore, Lucca, Italy \\ ${ }^{4}$ Department of Cardio-Thoracic-Vascular, Integrated Cardiology Services, Azienda Ospedaliera San Camillo Forlanini, \\ Rome, Italy \\ ${ }^{5}$ Department of Cardio-Thoracic-Vascular, Cardiology Unit, Azienda Ospedaliera San Camillo Forlanini, Rome, Italy \\ ${ }^{6}$ Department of Clinical and Rehabilitation Cardiology, Presidio Ospedaliero San Filippo Neri, ASL Roma 1, Rome, Italy \\ ${ }^{7}$ Department of Cardiology, Azienda di Rilievo Nazionale e Alta Specializzazione Garibaldi, Catania, Italy \\ ${ }^{8}$ Fondazione per il Tuo cuore - Heart Care Foundation, Florence, Italy
}

\section{CORRESPONDING AUTHOR:}

Luigi Tarantini

Department of Cardiology

Presidio Ospedaliero - Santa Maria Nuova, AUSL RE IRCCS

viale Risorgimento 80

42123 Reggio Emilia, Italy

E-mail: luigi.tarantini@gmail.com; luigi.tarantini@ausl.re.it

ORCID: 0000-0003-2580-0963

Doi: $10.48286 /$ aro.2021.27

History

Received: Sept 16, 2021

Accepted: Nov 11, 2021

Published: Dec 1, 2021

\section{ABSTRACT}

Covid-19 pandemic has completely overthrown health system organization but at the same time it has provided the proof that the patterns of care could rapidly adapt to new circumstances. Social distancing and limited access to hospitals have proven to be a valid tool to reduce Sars-Cov2 infection but they could be useful to control many other infective diseases, especially for frail patients as people with cancer are. In a model of chronic care for long-term cancer patients and/or within a survivorship program for cured patients, Cardioncology should built upon the experience of Covid pandemic and set new management strategies to provide top level care for a wide and increasing patient population. Telemedicine, patients and caregivers empowerment together with dedicated resources are mandatory to set a real change in cardiac care for cancer patients through and beyond pandemic. 


\section{KEY WORDS}

Cardioncology; Covid-19; cancer survivors; telemedicine.

\section{IMPACT STATEMENT}

The crisis caused by Covid-19 requires the remodeling of cardioncology in order to avoid the SarsCOV-2 infection. Telemedicine and the re-engineering of management algorithms can represent a valid tool for the prevention and treatment of cardiotoxicity.

fection leads to secondary hemophagocytic lymph histiocytosis (sHLH), which is a multiorgan hyperinflammatory condition based on the hyperactivation of cytotoxic T lymphocytes, macrophages, and natural killer cells, leading to multiorgan failure (including myocarditis, venous thromboembolism, and acute respiratory distress syndrome) and consequently to death (9). However, the Covid-19 "crisis" can represent an opportunity to create new management strategies that can help overcome some problems that in the past prevented the full development and spread of Cardioncology programs.

f the Covid-19 disease caused a significant stress to health systems organization as entire hospitals or hospital wards have been shifted towards the exclusive treatment of the disease so limiting the available resources dedicated to the management of other diseases. Consequently covid-19 pandemic had significant impact on the management of illness not directly connected with Sars-Cov2 infection. Even in March 2021, for example, in Piedmont an important region in Northern Italy, all elective hospital admissions not directly connected with covid-19 have been suspended again for the spread of the third epidemic wave (1). During the first phase of the Covid-19 pandemic, we have witnessed an increased incidence of patients with complicated or "delayed" acute myocardial infarction presentation (2) and the same occurred for heart failure (3). In clinical oncology practice the Covid-19 pandemic had significant negative effects reducing screening activities and oncological surveillance programs (4-7) with a possible increase of cancer mortality $(7,8)$. For such reasons, uncertainty in predicting when we will overcome the Covid-19 crisis imposes substantial reflection also in Cardioncology practice to prevent and effectively treat cardiotoxicity. Indeed, patients with active cancer or those treated with cardiotoxic therapies may have heart damages exacerbated by SARS-CoV-2 infection than non-cancer patients. SARS-CoV-2 in-

\section{CARDIONCOLOGY: THE NEW MAGMATIC AND MOVING SUB- SPECIALIZATION OF CARDIOLOGY}

Patients with cancer often have coexisting cardiovascular (CV) risk factors that must be appropriately managed and followed even in the medium to long-term considering the success of current oncological therapies $(10,11)$. The new therapies introduced for the treatment of cancer in recent years, indeed, if on the one hand has revolutionized and improved the prognosis of many neoplasms, on the other hand can cause a wide spectrum of short- and long-term cardiotoxic effects beyond heart failure. A practical example is represented by the need to check the ECG, blood pressure, lipid, glycemic homeostasis in patients on treatment with drugs able to lengthen the QT or for those which interfere with vascular/metabolic homeostasis (12) to prevent arrhythmias or cardiac ischemic complications. For a successful management program, a fundamental point in the evolving field of Cardioncology is the collaboration and sharing of multi-specialist skills during all phases of the cancer therapeutic program (figure 1) $(13,39)$. The 
improvement in patient survival resulted in the birth of a new and growing category of "long-living" cancer patients with the need for multidisciplinary periodic checks to avoid late cardiac complications due to progressive myocardial dysfunction or accelerated atherosclerosis. This is also (and especially) true for patients with metastatic cancer in ongoing therapy. Currently, the management of oncological disease in networked hospitals can pose logistical problems related to patient transfers and the relocation of specialist structures to different hospitals. Nowadays the daily calendar of cancer patients in active treatment is full of commitments and hospital contacts between blood samples, imaging tests, radiotherapy session, cyclic therapy infusions and set "timely" scheduled controls of different specialties, as cardiological evaluation and relative diagnostic tests, could represent a problem overall for patients who live far from reference centers or for those who present a condition of disability and non-self-sufficiency. These logistical (and organizational) aspects, a real problem to implement cardioncology programs in some centers in pre covid-19 time, became even more important and generalized in the period of the Covid-19 pandemic due to the limitation or deletion of scheduled hospital visits to limit the exposure of patients and health- care professionals. Furthermore, social distancing and limitation to access had a negative influence also for rehabilitation programs and therefore prevented the development or continuation of prevention and rehabilitation programs, one of the most promising sections of cardioncology overall in the field of long-living cancer patients $(14,15)$.

The central question that the covid-19 crisis forces us to ask ourselves is therefore: how can we create an effective and efficient management program in Cardioncology while maintaining social distancing, limiting access to the hospital and possibly not increase the financial costs of management?

\section{TELEMEDICINE: FROM SOLUTION DURING PANDEMIC TO VALID OPPORTUNITY FOR THE DEVELOPMENT OF CARDIONCOLOGY BEYOND COVID-19}

Covid-19 outbreak "scenario" gave impetus to development and diffusion of telemedicine $(16,17)$, a practice that allows to maintain contact with the patient and at the same time respect the distance, thus reducing the risk of infection. The progression

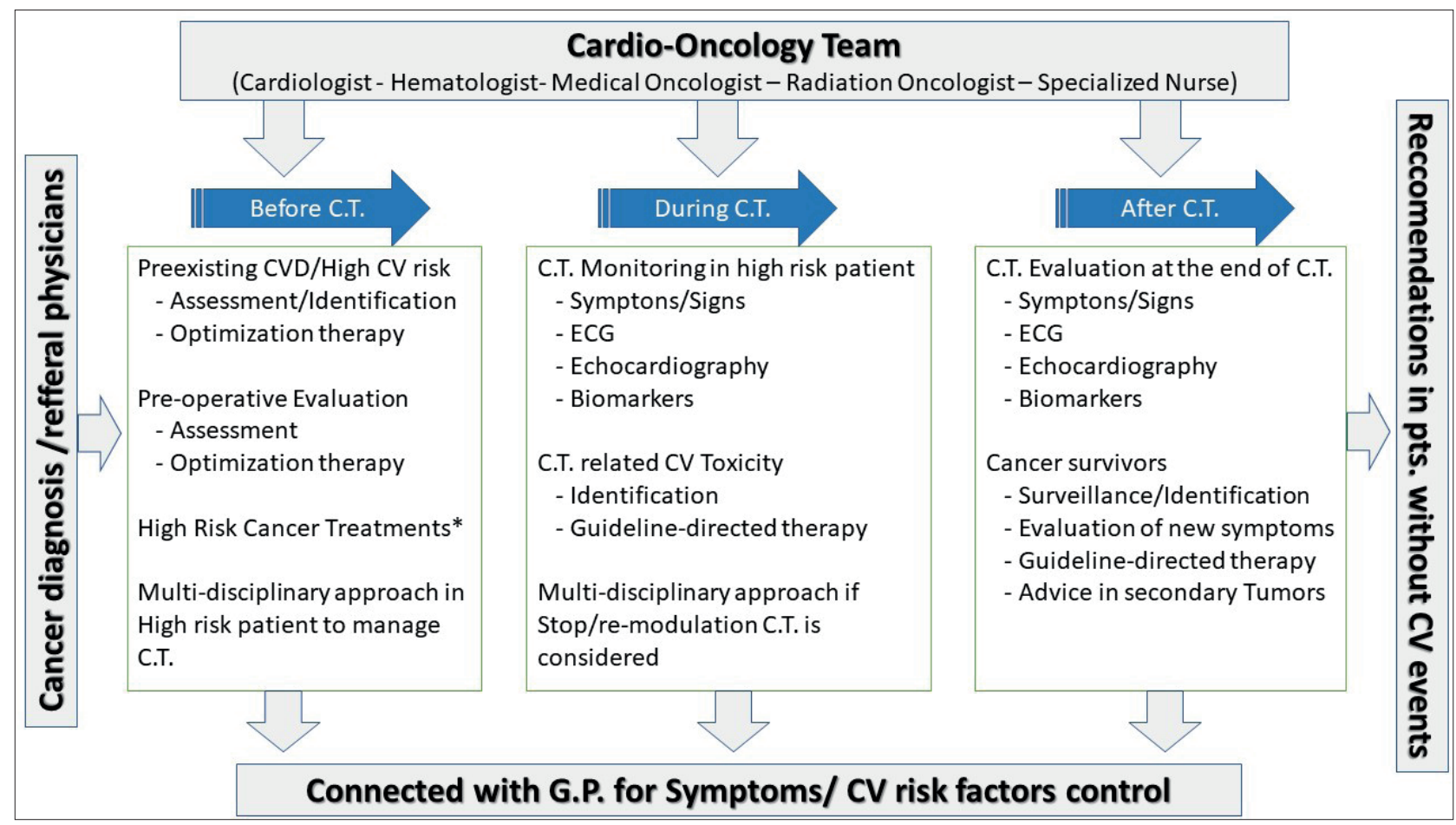

Figure 1. Cardio-oncology: rationale and pathways for cancer patients. Modified by Lancellotti (13).

C.T.: Cancer Treatments; CVD: Cardiovascular disease; CV: cardiovascular; ECG: electrocardiogram; *: Anthracyclines, anti-HER2 therapies, VEGF or BCR-ABL targeted TKIs, proteosomal inhibitors, and thoracic radiotherapy. TKI: tyrosine kinase inhibitor; VEGF, vascular endothelial growth factor 
towards electronic health records has facilitated the sharing of information between different categories of specialists involved in patient care and in different settings (hospital and outpatients) hence improving continuity of care. Virtual platforms have proved useful instruments for multidisciplinary discussion and video consultation with staff involved in patient care, with the patient himself or caregiver in family environment. The spread of telemonitoring employing mobile phones allows us remote control of useful parameters such as oxygen saturation, blood pressure, ECG, glycemic values in diabetics. Since the beginning of the COVID-19 pandemic, several initiatives have been launched on the use of telemedicine to check and control atrial fibrillation (18), diabetes (19), hypertension (20), and heart failure (21-23), clinical conditions common also in the cardioncology practice. Preliminary clinical experience suggests the technological improvement may support also the synchronous telerehabilitation programs (24). Finally, social media represent one potential opportunity to disseminate information about cardio-oncology promoting educational/advocacy campaign to a large audience (25). Another remark makes telemedicine appealing, COVID-19 "crisis" has also severely affected the global economy. Significant reductions in income, a rise in unemployment, and disruptions in the transportation, service, and manufacturing industries are among the consequences of the Covid-19 pandemics the effects of which will be increasingly relevant as the epidemic continues and likely will have repercussions for a long time after we emerge from the current situation of uncontrolled covid-19 outbreak. The economic consequences will be amplified by heterogeneous income distribution across the country causing a potential inequality in care and consequently a loss of effectiveness and efficiency of health systems including universalistic ones (26). Social and economic factors are emerging as relevant factors conditioning the prognosis of cancer $(27,28)$ and heart disease $(29,30)$, real-world evidence underscores the importance of not neglecting these factors also in cardioncology $(31,32)$. A "syndemic" approach tacking in consideration the intimate intersection between clinical, economic, and social factors could help to face "complex" situation as often happens to meet in the practice of cardioncology. By preserving interpersonal connectivity and therefore reducing distances, telemedicine can mitigate social/geographic isolation, improve the circulation of information, and most probably reduce the costs deriving from saving time and money for transport. The routine video consultation aimed at not losing contact with the patient can also be performed by dedicated nursing staff who, in addition to having a positive effect on compliance and adherence to clinical recommendations and prevention programs, can operate as a "navigator" to select patients who need traditional "faceto-face" clinical visits. In order to improve patients' compliance to both virtual and in-person activities, a personalized empowerment program should be proposed. Caregivers (mainly within the family unit) play a pivotal role in such process and should consequently be part of this program (33).

\section{IN SEARCH OF THE "HOLY GRAIL": DEVELOP AND IMPLEMENTING CARDIONCOLOGY AFTER THE COVID-19 CRISIS}

The main purpose of Cardioncology is to assists in the overall care of cancer patients, with and without cardiovascular disease, in an interdisciplinary way sharing responsibilities and experiences among health-care team members to reduce cancer therapeutics-related cardiovascular complications and improve clinical outcomes. This collaborative model results in completion of cancer therapy in most patients (34-37) nevertheless we are still far from having established reference standards on the structural level $(38,39)$ and a discrepancy in awareness of the problem of cardiotoxicity persists (40). In recent years, international scientific societies have attempted to fill these gaps both on an organizational (13) and scientific level $(9,41-43)$. However, it is worth remembering there are currently no established benchmarks to guide clinicians regarding timely access and assessment of patients. Cardioncology is not bound by a traditional and inflexible patient-care relationship. Patients receiving active cancer treatment generally require a faster access for basal evaluation with periodic hospital-based surveillance check-up during the completion of therapeutic program. Important variables which influence the frequency of checks are the potential cardiotoxicity of agents, the clinical status of patients including cardiac history and presence/absence of comorbidity. Over the years, combined with this "traditional" activity, the progressive increase in long-lived cancer patients has led to the creation of some outpatient clinics dedicated to the surveillance of late cardiot- 
oxic effects and to the supervision of subjects in continuous palliative cancer treatment in many centers. Covid-19 outbreak heavily conditioned the cardioncology routine care in relation to the concern about Sars-COV2 infection, the measure for quarantine isolation and the inevitable reallocation of medical resources. Specific scenarios and algorithms for cardioncology have been suggested to manage patient during the outbreak of covid-19 (44-47) aimed to secure a separate and protected access to oncology, hematology and cardio-oncology departments and clinics without compromise the cardioncology consultation. These proposals have in common some key points as the accurate risk identification at basal evaluation of patients, a more intensive use of telehealth, a more stringent use of traditional hospital-based imaging assessments to be integrated with alternative methods such as biomarkers, easier to perform during periodic follow-up blood tests or drug infusion sessions. Preliminary experiences indicate that the remodeling of cardioncology activity through the integration of traditional "in-person" and "virtual" telemedicine care is achievable (47). Although we do not have the comparison results with respect to the traditional management method, it is reasonable to assume that in the future we will have to reformulate our way of working (table I) and that this is a way to be pursued for at least three reasons:

1. the lesson we have learned from Covid-19 is that our globalized society, interconnected and with a growing economic/social gap causes different responses from different healthcare systems, favoring the spread of infectious diseases such as those caused by highly infectious Sars-Cov2 virus capable of mutate rapidly. The "wave" course of Covid-19 requires the creation of flexible management models capable to rapidly respond to and contain the outbreak through isolation and social distancing without losing contact with patients and compromising the effectiveness of cancer treatments.

2. The Covid-19 outbreak has caused the cancellation of many scheduled visits with most of them should be reprogrammed. Wave trend of the COVID-19 epidemic with periodic remissions and resurgence impose the maintenance of safety protocols such as the separation of Covid hospitals from the Covid-19 "free" ones, or the extension of the time of medical and sanitary services to maintain social distancing and to allow the sanitation of environments and equip- ment. It will be exceedingly difficult reabsorb in a reasonable time the missed controls and, at the same time, guarantee an appropriate and "timely" management of new cases.

3. In the last few years there has been a real revolution in the field of oncological treatments, new drugs have been launched and innovative therapies have been developed such as immunotherapy with immune-check point inhibitors and Car-T. Cardiological surveillance is often required in many of these cases, thus expanding the horizon and the volume of activity of cardioncology clinics. There is, therefore, a need to reshape the surveillance and care pathways to avoid the repetition of unnecessary visits and examinations without denying specialist cardiological support and losing contact with the patient. Such consideration is not trivial considering the increase of patients who respond favorably to cancer treatments and at risk of cardiotoxicity in a large group of individuals such as the elderly or frail patients.

However, the path is not easy, there are many challenges to be faced and problems to be solved. A first necessary consideration is related to telemedicine, which still requires an enhancement in its diffusion, in the definition and homologation in the standards, the clarification of the legal terms and finally the reimbursement and professional recognition. There are other aspects to consider regarding telemedicine. The cost of the equipment and the ability to use new technologies can be a problem for the application of this method in some groups of patients, a second, and no less important consideration is that it must be an integrative method and not a substitute for traditional approach. The crisis caused by Covid-19 has also had a significant impact on mental health and psychological balance (48), a relevant issue in patients with cancer. The remote contact with the use of telemedicine is useful for maintaining distance but is less effective for the emotional effects of isolation which in some cases can also be amplified if used as an alternative and not integrative method of the traditional "face to face" approach. A judicious and flexible use is therefore advisable, in order to avoid a sense of abandonment and detachment for patients.

Another relevant issue concerns the use of biomarkers. Troponin or NT-proBNP (N-terminal proB-type natriuretic peptide), may be able to allow spacing out of the serial echocardiograms, which are standard cardio-oncology practice. Biomarkers 
Careful history and physical examination of cancer patients in order to intercept moderate to high-risk pts for cardiotoxicity (i.e. previous heart diseases, two or more CV risk factors, previous cardiotoxic treatment, history of cardiotoxicity) - Before cancer treatment (anticancer drugs, radiotherapy and cancer surgery): optimize cardiovascular treatment, review drug-drug interactions, stablish follow-up needs avoiding

Objectives futile in-person visit

- During cancer treatment: monitoring of cancer treatments favoring focused echocardiography and biomarkers as "screening" tools for early diagnosis and treatment of CV complications in asymptomatic pts.

- Long-term survivors surveillance using the devices of telemedicine to be integrated into a "hybrid" system on a personalized basis focused on the characteristics of the patient

$\begin{array}{ll}\text { Personel } & \begin{array}{l}\text { Cardio-oncologist } \\ \text { Specialized nurse }\end{array} \\ \text { Equipment } & \begin{array}{l}\text { Sphygmomanometer } \\ \text { ECG including QTc measurement } \\ \text { Transthoracic echocardiography (TTE) } \\ \text { Portable TTE (focused echocardiography) }\end{array} \\ & \begin{array}{l}\text { Lack of consensus or agreement among the } \\ \text { participants in multi-disciplinary management } \\ \text { program. } \\ \text { Obstacles of professional training and professional } \\ \text { selection }\end{array}\end{array}$

Patients with low risk of developing cardiotoxicity

From cancer specialists to cardiologists - Optimization of cardiovascular risk factors control in asymptomatic patients without structural heart disease

- Advice regarding drug-drug interactions

- Advice regarding referral for re-evaluation or referral for in-depth diagnostic or therapeutic cardiological procedures in centers with major experience

From GPs' to cardiologists

- Advice regarding referral to cardio-oncology clinic From patients to specialized nurses (e-nurse cardio-oncology consult)

- Tight controls of some patients after C-O consultation focused on diet advice, exercise recommendations, new symptoms, treatment tolerance, treatment adherence, blood pressure, or glucose control

Cardio-oncologist Specialized nurse

Digitalized medical records

Cardiac remote monitoring (i.e. blood pressure, ECG)

Biomarkers (Troponin, NtProBNP, BNP)

Lack of homologation and standardization.

Uncertainty of legal coverage, Lack of professional recognition and financial reimbursement

Lack of professional training and professional selection

Table I. Re-engineering cardioncology in "Covid-19 Age". Modified by Lancellotti (13).

CV: Cardiovascular; GPs: General Practitioners; C-O: Cardio-oncology; ECG: Electrocardiogram; BNP: Brain Natriuretic Peptide; NTProBNP: Amino terminal fragmente of BNP. .

are relatively lower cost, lower risk, less invasive, and less COVID-19 exposure approach compared with imaging. Nevertheless, currently we do not have "solid" evidence available from cardiotoxicity surveillance. Moving to a primarily biomarker-based monitoring could compromise sensitivity and specificity of cardiotoxicity detection. Further research is needed on biomarkers to detect cardiotoxicity. This strategy can be considered on an individual basis, balancing risk of COVID-19 exposure and the patient's cardiotoxicity risk. Integration methods between biomarkers and imaging tests can be implemented such as performing "fast" echocardiogram focused on the ejection fraction by organizing the examination in appropriate spaces during access for oncological therapy sessions, or where feasible in sessions home assistance.

In conclusion, the Covid-19 pandemic, with more than 225 million cases and over 4.5 million deaths worldwide today (49), has upset our way of living and working and its effects will also extend for a long time once the pandemic has passed. It is difficult to hypothesize a return, at least in the short term, to the old organizational and clinical practice models (50) and cardioncology will be one of the subspecialty branches of cardiology that will be primary involved. Despite the initial crisis we are learning to react, and the era of COVID-19 is teaching us new paradigms of medicine that will change the face of medical practice. Now has come the time to apply what we have learned in past in cardio-cancer in a more effective, easy, flexible, and far-reaching way.

\section{ETHICS}

\section{Fundings}

There were no institutional or private fundings for this article. 


\section{Conflict of interests}

The authors have declared no conflict of interests.

\section{REFERENCES}

1. Available from: https://www.ansa.it/piemonte/notizie/2021/03/10/piemonte-sospende-ricoveri-no-covid-in-tutti-gli-ospedali_467f97199637-481c-8978-3c6b51af9a62.html. Last access: Sept 13, 2021.

2. De Rosa S, Spaccarotella C, Basso C, et al. Reduction of hospitalizations for myocardial infarction in Italy in the COVID-19 era. Eur Heart J 2020;41(22):2083-88.

3. Cannatà A, Bromage DI, Rind IA, et al. Temporal trends in decompensated heart failure and outcomes during COVID-19: a multisite report from heart failure referral centres in London. Eur J Heart Fail 2020;22(12):2219-24.

4. Morris EJA, Goldacre R, Spata E, et al. Impact of the COVID-19 pandemic on the detection and management of colorectal cancer in England: a population-based study. Lancet Gastroenterol Hepatol 2021;6(3):199-208.

5. Dinmohamed AG, Visser O, Verhoeven RHA, et al. Fewer cancer diagnoses during the COVID-19 epidemic in the Netherlands. Lancet Oncol 2020;21(6):750-1.

6. Peacock HM, Tambuyzer T, Verdoodt F, et al. Decline and incomplete recovery in cancer diagnoses during the COVID-19 pandemic in Belgium: a year-long, population-level analysis. ESMO Open 2021;6(4):100197. Doi: 10.1016/j. esmoop.2021.100197.

7. Maringe C, Spicer J, Morris M, et al. The impact of the COVID-19 pandemic on cancer deaths due to delays in diagnosis in England, UK: a national, population-based, modelling study. Lancet Oncol 2020;21(8):1023-34.

8. Hanna TP, King WD, Thibodeau S, et al. Mortality due to cancer treatment delay: systematic review and meta-analysis. BMJ 2020;371:m4087.

9. Quagliariello V, Bonelli A, Caronna A, et al. SARSCoV-2 Infection and Cardioncology: From Cardiometabolic Risk Factors to Outcomes in Cancer Patients. Cancers (Basel) 2020;12(11):3316. Doi: 10.3390/cancers12113316.

\section{Authors' contribution}

All the authors contributed equally to conception, data collection, analysis and writing of this paper.

10. Curigliano G, Lenihan D, Fradley M, et al. ESMO Guidelines Committee. Electronic address: clinicalguidelines@esmo.org. Management of cardiac disease in cancer patients throughout oncological treatment: ESMO consensus recommendations. Ann Oncol 2020;31(2):171-90. Doi: 10.1016/j.annonc.2019.10.023.

11. Armenian $\mathrm{SH}, \mathrm{Xu} \mathrm{L}, \mathrm{Ky} B$, et al. Cardiovascular Disease Among Survivors of Adult-Onset Cancer: A Community-Based Retrospective Cohort Study. J Clin Oncol 2016;34(10):1122-30. Doi: 10.1200/JCO.2015.64.0409.

12. Campia U, Moslehi JJ, Amiri-Kordestani L, et al. Cardio-Oncology: Vascular and Metabolic Perspectives: A Scientific Statement From the American Heart Association. Circulation 2019;139(13):e579-e602. Doi: 10.1161/ CIR.0000000000000641. Erratum in: Circulation 2019;139(15):e838-e839.

13. Lancellotti $P$, Suter TM, López-Fernández $T$, et al. Cardio-Oncology Services: rationale, organization, and implementation. Eur Heart J 2019;40(22):1756-63.

14. Brown SA. Preventive Cardio-Oncology: The Time Has Come. Front Cardiovasc Med. 2020;6:187. Doi: 10.3389/fcvm.2019.00187.

15. Venturini $E$, lannuzzo G, D'Andrea A, et al. Oncology and Cardiac Rehabilitation: An Underrated Relationship. J Clin Med 2020;9(6):1810. Doi: 10.3390/jcm9061810.

16. Shaw JG, Sankineni S, Olaleye CA, et al. A Novel Large Scale Integrated Telemonitoring Program for COVID-19. Telemed J E Health 2021. Doi: 10.1089/tmj.2020.0384.

17. Agarwal P, Mukerji G, Laur C, et al. Adoption, feasibility and safety of a family medicine-led remote monitoring program for patients with COVID-19: a descriptive study. CMAJ Open. 2021;9(2):E324-E330. Doi: 10.9778/ cmajo.20200174. PMID: 33795222.

18. Gawałko $M$, Duncker $D$, Manninger $M$, et al. The European TeleCheck-AF project on remote appbased management of atrial fibrillation during 
the COVID-19 pandemic: centre and patient experiences. Europace 2021 Apr 2: euab050. Doi: 10.1093/europace/euab050.

19. Luzi L, Carruba M, Crialesi R, et al. Telemedicine and urban diabetes during COVID-19 pandemic in Milano, Italy during lock-down: epidemiological and sociodemographic picture. Acta Diabetol 2021:1-9.

20. Omboni S, McManus RJ, Bosworth HB, et al. Evidence and Recommendations on the Use of Telemedicine for the Management of Arterial Hypertension: An International Expert Position Paper. Hypertension 2020;76(5):1368-83.

21. Salzano A, D'Assante R, Stagnaro FM, et al. Heart failure management during the COVID-19 outbreak in Italy: a telemedicine experience from a heart failure university tertiary referral centre. Eur J Heart Fail 2020;22(6):1048-50.

22. D'Amario D, Restivo A, Canonico F, et al. Experience of remote cardiac care during the COVID-19 pandemic: the V-LAP ${ }^{\text {TM }}$ device in advanced heart failure. Eur J Heart Fail 2020;22(6):1050-2.

23. Jiang $X$, Yao J, You JH. Cost-effectiveness of a Telemonitoring Program for Patients with Heart Failure During the COVID-19 Pandemic in Hong Kong: Model Development and Data Analysis. J Med Internet Res 2021;23(3): e26516. Doi: 10.2196/26516.

24. Zhu S, Zhang L, Xie S, et al. Reconfigure rehabilitation services during the Covid-19 pandemic: best practices from Southwest China. Disabil Rehabil 2021;43(1):126-32. Doi: 10.1080/09638288.2020.1853828.

25. Conley CC, Goyal NG, Brown SA. \#CardioOncology: Twitter chat as a mechanism for increasing awareness of heart health for cancer patients. Cardiooncology 2020;6:19. Doi: 10.1186/ s40959-020-00072-w.

26. Pak A, Adegboye OA, Adekunle Al, Rahman KM, McBryde ES, Eisen DP. Economic Consequences of the COVID-19 Outbreak: the Need for Epidemic Preparedness. Front Public Health 2020;8:241. Doi: 10.3389/fpubh.2020.00241.

27. Unger JM, Moseley AB, Cheung CK, et al . Persistent Disparity: Socioeconomic Deprivation and Cancer Outcomes in Patients Treated in Clinical Trials. J Clin Oncol 2021:JCO2002602. Doi: 10.1200/JCO.20.02602.

28. Kurani SS, McCoy RG, Lampman MA, et al. Association of Neighborhood Measures of Social Determinants of Health with Breast, Cervical, and Colorectal Cancer Screening Rates in the US
Midwest. JAMA Netw Open 2020;3(3):e200618. Doi:10.1001/jamanetworkopen.2020.0618.

29. Witte KK, Patel PA, Walker AMN, et al. Socioeconomic deprivation and mode-specific outcomes in patients with chronic heart failure. Heart 2018;104(12):993-8.

30. Havranek EP, Mujahid MS, Barr DA, et al. American Heart Association Council on Quality of Care and Outcomes Research, Council on Epidemiology and Prevention, Council on Cardiovascular and Stroke Nursing, Council on Lifestyle and Cardiometabolic Health, and Stroke Council. Social Determinants of Risk and Outcomes for Cardiovascular Disease: A Scientific Statement from the American Heart Association. Circulation 2015;132(9):873-98.

31. Batra A, Kong S, Cheung WY. Associations of Socioeconomic Status and Rurality With New-Onset Cardiovascular Disease in Cancer Survivors: A Population-Based Analysis. JCO Oncol Pract 2021;17(8):e1189-e1201. Doi: 10.1200/ OP.20.01053.

32. Appiah D, Farias RM, Olokede OA, et al. The influence of individual and neighborhood-level characteristics on rural-urban disparities in cardiovascular disease mortality among U.S. women diagnosed with breast and gynecologic cancers. Gynecol Oncol 2021;161(2):483-90. Doi: 10.1016/j.ygyno.2020.11.023.

33. Bisceglia I, Gabrielli D, Canale ML, et al. ANMCO POSITION PAPER: cardio-oncology in the COVID era (CO and CO). Eur Heart J Suppl. 2021;23(Suppl C):C128-C153. Doi: 10.1093/eurheartj/suab067.

34. Kappel C, Rushton M, Johnson C, et al. Clinical experience of patients referred to a multidisciplinary cardio-oncology clinic: an observational cohort study. Curr Oncol 2019;26(3): e322-e327. Doi: 10.3747/co.26.4509.

35. Sulpher J, Mathur S, Graham N, et al. Clinical Experience of Patients Referred to a Multidisciplinary Cardiac Oncology Clinic: An Observational Study. J Oncol 2015;2015:671232. Doi: 10.1155/2015/671232.

36. Pareek N, Cevallos J, Moliner P, et al. Activity and outcomes of a cardio-oncology service in the United Kingdom-a five-year experience. Eur J Heart Fail 2018;20(12):1721-31. Doi: 10.1002/ejhf.1292.

37. Costa IBSDS, Bittar CS, Fonseca SMR, et al. Brazilian cardio-oncology: the 10-year experience of the Instituto do Cancer do Estado de Sao 
Paulo. BMC Cardiovasc Disord 2020;20(1):206.

38. Canale ML, Lestuzzi C, Bisceglia I, et al. Cardio-oncology organization patterns in Italy: one size does not fit all. J Cardiovasc Med (Hagerstown) 2018;19(5):229-33. Doi: 10.2459/ JCM.0000000000000642.

39. Canale ML, Turazza F, Lestuzzi C, et al. Portrait of Italian Cardio-Oncology: Results of a Nationwide Associazione Nazionale Medici Cardiologi Ospedalieri (ANMCO) Survey. Front Cardiovasc Med 2021;8:677544. Doi: 10.3389/fcvm.2021.677544.

40. Peng J, Rushton M, Johnson C, et al. An international survey of healthcare providers' knowledge of cardiac complications of cancer treatments. Cardiooncology 2019;5:12.

41. Armenian SH, Lacchetti C, Barac A, et al. Prevention and Monitoring of Cardiac Dysfunction in Survivors of Adult Cancers: American Society of Clinical Oncology Clinical Practice Guideline. J Clin Oncol 2017;35(8):893-911. Doi: 10.1200/JCO.2016.70.5400.

42. Tarantini L, Gulizia MM, Di Lenarda A, et al. Documento di consenso ANMCO/AICO/AIOM: Snodi clinico-gestionali in ambito cardioncologico - ANMCO/AICO/AIOM Consensus document: Clinical and management pathways in cardio-oncology]. G Ital Cardiol (Rome) 2017;18(1):14-66.

43. Lyon AR, Dent S, Stanway S, et al. Baseline cardiovascular risk assessment in cancer patients scheduled to receive cardiotoxic cancer therapies: a position statement and new risk assessment tools from the Cardio-Oncology
Study Group of the Heart Failure Association of the European Society of Cardiology in collaboration with the International Cardio-Oncology Society. Eur J Heart Fail 2020;22(11):1945-60.

44. Calvillo-Argüelles O, Abdel-Qadir H, et al. Modified Routine Cardiac Imaging Surveillance of Adult Cancer Patients and Survivors During the COVID-19 Pandemic. JACC Cardio Oncol 2020;2(2):345-9.

45. Farmakis D, Keramida K, Filippatos G. Cardio-oncology services during the COVID-19 pandemic: practical considerations and challenges. Eur J Heart Fail 2020;22(6):929-32.

46. Addison D, Campbell CM, Guha A, et al. Cardio-Oncology in the Era of the COVID-19 Pandemic and Beyond. J Am Heart Assoc 2020;9(19):e017787.

47. Brown SA, Patel S, Rayan D, et al. A virtual-hybrid approach to launching a cardio-oncology clinic during a pandemic. Cardiooncology 2021;7(1):2.

48. Torales J, O' Higgins M, Castaldelli-Maia JM, Ventriglio A. The outbreak of COVID-19 coronavirus and its impact on global mental health. Int J Soc Psychiatry 2020;66(4):317-20

49. Available from: https://coronavirus.jhu.edu/ map.html. Last access: Sept 14, 2021.

50. Gupta AK, Jneid H, Addison D, et al. Current perspectives on Coronavirus 2019 (COVID-19) and cardiovascular disease: a white paper by the JAHAeditors.JAm HeartAssoc2020;9:e017013.v. Doi: 10.1161/JAHA.120.017013. 